\title{
Periodic Solutions for Stochastic Differential Equations Driven by General Counting Processes: Application to Malaria
}

\author{
KOUAME Yao Simplice ${ }^{1}$ and NZI Modeste ${ }^{1}$ \\ ${ }^{1}$ Mathematics and Computer Science Training and Research Unit, Felix Houphouet Boigny University of Cocody, 22 Bp \\ 582 Abidjan 22, Abidjan, Côte d'Ivoire \\ Correspondence: Modeste, N'ZI, Mathematics and Computer Science Training and Research Unit, Felix Houphouet \\ Boigny University of Cocody, 22 Bp 582 Abidjan 22, Abidjan, Côte d'Ivoire. E-mail: modestenzi@yahoo.fr
}

Received: March 29, 2021 Accepted: June 15, 2021 Online Published: June 22, 2021

doi:10.5539/jmr.v13n4p1 URL: https://doi.org/10.5539/jmr.v13n4p1

\begin{abstract}
In this paper, a class of periodic stochastic differential equations driven by general counting processes (SDEsGp) is studied. First, an existence-uniqueness result for the solution of general SDEsGp based on Poisson processes with $\tau$ periodic stochastic intensity of time $t$ has been given, for some $\tau>0$. Then, using the properties of periodic Markov processes, sufficient conditions for the existence and uniqueness of a periodic solution of the considered equations are obtained. We will then apply the obtained results to the propagation of malaria in a periodic environment.
\end{abstract}

Keywords: poisson process, stochastic differential equation, periodic solution, Markov process, epidemic model

\section{Introduction}

In stochastic modeling, a dynamical system is the set constituted by a system and stochastic evolution equations describing the random evolution of its trajectories. There are two types of systems in the literature: discrete systems and continuous systems. Discrete systems are generally described by jump processes which give a good representation of the studied phenomenon. Jump processes are stochastic counting processes $\left(J_{t}\right)_{t \geq 0}$ whose paths are constant except for jumps of size +1 or -1 . The simplest exemple is, of course the Poisson process $\left(P_{t}\right)_{t \geq 0}$ which attracted much attention due to its wide applications in the fields where counting problems arise: Queues, population dynamic, chimical reaction, pharmacology, biology, medical sciences, celestial mechanics, statistical physics, and so on . Note also that the distribution of this process is determined by specifying the intensity parameter $\lambda$ which gives

$$
\mathbb{P}\left(P(t+\Delta t)-P(t)>0 \mid \mathcal{F}_{t}\right)=\lambda \Delta t+o(\Delta t)
$$

where $\mathcal{F}_{t}$ is the history for the process up to time $t$. Usually, more general counting processes are determined by specifying a stochastic intensity function $\lambda(t, P)$ which as in Eq.(1), gives

$$
\mathbb{P}\left(P(t+\Delta t)-P(t)>0 \mid \mathcal{F}_{t}\right)=\lambda(t, P) \Delta t+o(\Delta t) .
$$

A number of authors have used this more generalized counting process to study stochastic differential equations of type;

$$
X(t)=X(0)+\sum_{\ell=1}^{k} h_{\ell} P_{\ell}\left(\int_{0}^{t} \lambda_{\ell}(s, X(s)) d s\right),
$$

where $X(t)=\left(X^{i}(t)\right), \quad \mathrm{i}=1,2, \ldots, d$ is a $d$-vector composed of jump processes and gives the state of the process at any time $t$ and the integer $k$ the total number of Poisson processes in the model.

For all $\ell$ and $\ell^{\prime}$ such that $\ell \neq \ell^{\prime}$, the stochastic processes $\left(P_{\ell}(t)\right)_{t \geq 0}$ and $\left(P_{\ell^{\prime}}(t)\right)_{t \geq 0}$ are independent standard Poisson processes. The theoretical development of stochastic process based on Poisson processes has be seen in recent years and several results have been proven: Existence and uniqueness of the solution of Eq.(3) are proved under more general conditions (Anderson, D.F and Kurtz, T.G, 2015), (Guy,R and all, 2014), (Guy,R and all, 2014). This result is discussed in more detail in Kurtz (Kurtz, T.G, 1980). In the above equations, the general counting process

$$
X_{\ell}(t)=P_{\ell}\left(\int_{0}^{t} \lambda_{\ell}(s, X(s)) d s\right)
$$


counts the jumps of type $\ell$ and $h_{\ell}$ gives the direction.

Taking expectation of the previous stochastic Eq.(3), we have the associate deterministic equation

$$
x(t)=x(0)+\int_{0}^{t} b(s, x(s)) d r
$$

where $b(t, x(t))=\sum_{\ell=1}^{k} h_{\ell} \lambda_{\ell}(t, x(t)) d t$. The study of Eq.(3) exhibe a great difficulty, which are caused by the jumps of Poisson processes random mesures when intensity functions are stochastic processes. As far as we know, there are no results on the existence and uniqueness of a periodic solutions for Eq.(3).

Here we study a stochastic differential equation of type Eq.(3) under the following assumptions:

For all $\ell \in \mathbb{N}, \lambda_{\ell}:\left[0,+\infty\left[\times\left(J\left[0,+\infty[)^{d}\right.\right.\right.\right.$ ( $d$ may be finite) is a Borel-mesurable function, where $J([0,+\infty[)$ denote the space of all functions that are rigth-continuous with left limits (cadlàg functions) such that $X(0)=X_{0} \neq 0$ and $X(t)-X\left(t^{-}\right) \in\{0,+1,-1\}$. In particular, if $X_{0}=0, X(t)=0$, for $t>0$. We define on $J([0,+\infty[)$ the Skorohod topology when a topology is needed (Billingsley, $\mathrm{P}, 1999$ ), (Lindvall, T, 1973). A trajectory $X(t, \omega)$ is said to be explodes in a bounded interval $[0, T]$ for some real $T>0$ if for any integer $K>0$, there exists a time $s \in[0, T]$ such that $|X(s, \omega)| \geq K$.

Let $\beta_{K}(X)$ denote the explosion time of the jump process $(X(t))_{t \geq 0}$. In the present work, we are interested in the existence result of the solution of Eq.(3). Now we introduce the assumptions $\left(H_{1}\right)-\left(H_{2}\right)$ which ensure the problem is well posed in our discussion.

- Assumption $1\left(\mathbf{H}_{1}\right)$ For all $X \in J\left(\left[0,+\infty[)^{d}\right.\right.$ and each $\ell$, we make those assumptions, we define a positive continue function $\tilde{\lambda}_{\ell}(t, x)$, which is $\tau$-periodic in time $t$ for some $\tau>0$, that is for all $t \geq 0$

$$
\tilde{\lambda}_{\ell}(t+\tau, X(t))=\tilde{\lambda}_{\ell}(t, X(t)), \text { for some } \tau>0,
$$

This assumption describes the impact of the variability of an environment on the intensity of the Poisson process, which will allow for periodic fluctuations in the environment on the dynamics of the system,

- Assumption $2\left(\mathrm{H}_{2}\right)$

$$
\int_{0}^{\beta_{K}(X)} \sum_{\ell=1}^{k} \tilde{\lambda}_{\ell}(t, X(t)) d t<\infty
$$

where

$$
\beta_{K}(X)=\inf \{t \geq 0,|X(t)| \geq K\}
$$

and $|X(t)|=\sum_{i=1}^{d}\left|X^{i}(t)\right|$

In the remainder of our work, we now pay attention to this stochastic differential equation (SDEsGp),

$$
X(t)=X(0)+\sum_{\ell=1}^{k} h_{\ell} P_{\ell}\left(\int_{0}^{t} \tilde{\lambda}_{\ell}(s, X(s)) d s\right) .
$$

Eq.(6) is equivalent to the system of $d$ equations

$$
X^{i}(t)=X^{i}(0)+\sum_{\ell=1}^{k} h_{\ell}^{i} P_{\ell}\left(\int_{0}^{t} \tilde{\lambda}_{\ell}^{i}(s, X(s)) d s\right), i=1, \ldots . d
$$

where $h_{\ell}^{i} \in\{0,-1,1\}$.

In the first part of this article, we give the results of existence and uniqueness of the solution for more general (SDEsGp) type. Next, we propose sufficient conditions under which we prove the existence and uniqueness of periodic solution to 
Eq.(6). Finally, an example is provided to demonstrate the obtained results and apply the previous analytical results to study stochastic dynamic for malaria epidemic model under periodic environment.

\section{Existence, Uniqueness of a Periodic Solution}

\subsection{Existence and Uniqueness}

Existence and uniqueness of the solution to Eq.(6) follows by using $\left(\mathrm{H}_{1}\right)$ and $\left(\mathrm{H}_{2}\right)$ to solve this equation "from one jump to the next". In Kurtz (Kurtz, T.G.,1982), the uniqueness of Eq.(4) implies $\int_{0}^{t} \lambda_{\ell}(s, X(s)) d s$ is a stopping time for $\left(X_{\ell}(t)\right)_{t \geq 0}$. Let $\mathcal{F}_{t}=\sigma\{X(s), 0 \leq s \leq t\}$ be the history for the stochastic process $(X(t))_{t \geq 0}$ up to time $t$ or the natural filtration for the process. Define by $\mathcal{M}_{\ell}(t)=P_{\ell}\left(\int_{0}^{t} \tilde{\lambda}_{\ell}(s, X(s)) d s\right)$, then by random time change properties the process $P_{\ell}(t)=\mathcal{M}_{\sigma_{\ell}(t)}$ is a standard Poisson process where $\sigma_{\ell}(t)=\inf \left\{s>0, \int_{0}^{s} \tilde{\lambda}_{\ell}(r, X(r)) d r>t\right\}$. We associate to the considered stochastic process $\left(\mathcal{M}_{\ell}(t)\right)_{t \geq 0}$ the process with random time change follow

$$
Q_{\ell}\left(\int_{0}^{t} \tilde{\lambda}_{\ell}(s, X(s)) d s\right)=\mathcal{M}_{\ell}(t)-\int_{0}^{t} \tilde{\lambda}_{\ell}(s, X(s)) d s .
$$

Therefore, Eq.(6) can be rewritten like the following equation

$$
X(t)=X(0)+\sum_{\ell=1}^{k} h_{\ell} Q_{\ell}\left(\int_{0}^{t} \tilde{\lambda}_{\ell}(s, X(s)) d s\right)+\int_{0}^{t} F(s, X(s)) d s,
$$

where $F(t, X(t))=\sum_{\ell=1}^{k} h_{\ell} \tilde{\lambda}_{\ell}(t, X(t))$.

The Eq.(9) is equivalent to the following equation

$$
X^{N}(t)=X^{N}(0)+\sum_{\ell=1}^{k} \frac{h_{\ell}}{N} Q_{\ell}\left(\int_{0}^{t} \tilde{\lambda}_{\ell}\left(s, N X^{N}(s)\right) d s\right)+\frac{1}{N} \int_{0}^{t} F\left(s, N X^{N}(s)\right) d s,
$$

where $N$ is a scale parameter and $X^{N}(t)=\frac{1}{N} X(t)$ is the vector of proportions. In a case of models with fixe total population size, $N=\sum_{l=1}^{d} X^{i}(t)$ is the total population size at time $t$. Then, $X^{N}(t)$ is the fractions vector of rescaled numbers of individuals in the various states at time $t$.

Proposition 2.1 Since $P_{\ell}(t)-t$ is a martingale, then the normalized process

$$
\tilde{Q}_{\ell}^{N}(t)=\frac{1}{N} Q_{\ell}\left(\int_{0}^{t} \tilde{\lambda}_{\ell}\left(s, N X^{N}(s)\right) d s\right) .
$$

is a $\mathcal{F}_{t}$-martingale and verifies

$$
\lim _{N \rightarrow \infty} \sup _{0 \leq r \leqslant T \wedge \beta_{K}(X)}\left|\tilde{Q}_{\ell}^{N}(r)\right| \rightarrow 0 \text { a.s ; for all } T>0 .
$$

Proof See Proposition 2.2.5 and Proposition 2.2.6 in T. Britton and E. Pardoux (Britton, T. and Pardoux, E., 2019).

Moment estimate is the most basic and useful technic of analyzing dynamic behavior of solution of stochastic functional differential equation. To study the moment estimate for studying stability (Mao, X.R., 2007), boundedness (Luo, Q. and all, 2011), existence and uniqueness of the solution to Eq.(6), we need the following Lemmas. 
Lemme 2.2 (Teng, L.Y, 2014) Let $(X(t))_{t \in[0, T]}$ be solution of the Eq.(6) if it exists. Then for $p \geq 0$, we have

$$
\left[\sup _{0 \leq r \leq T}|X(r)|\right]^{p}=\sup _{0 \leq r \leq T}|X(r)|^{p}
$$

Lemme 2.3 Assume that there is a function $L(t) \in L^{1}\left([0, T] ; \mathbb{R}_{+}\right)$such that $L(t)<\infty$ for any given $t \in[0, T]$, where $T>0$ and $F(t, x)$ verifies

$$
|F(t, x)-F(t, y)| \leq L(t)|x-y|
$$

for all $t \in[0, T], x, y \in \mathbb{R}^{n}$,

$$
F(t, 0) \in L^{1}\left([0, \tau], \mathbb{R}^{n}\right),
$$

and there exists $p \geq 2$ such that $\lim _{N \rightarrow \infty} \mathbb{E}\left(\left|\tilde{Q}_{\ell}^{N}(t)\right|^{p}\right)<\infty$ for each $t \in[0, T]$. Then there is a unique solution $(X(t))_{t \in[0, T]}$ to Eq.(6) and $\mathbb{E}\left[\sup _{0 \leq r \leq t}|X(r)|^{p}\right]<\infty$ for each $t \in[0, T]$.

Proof Set $X_{0}=X(t=0)$ the initial value of the process $(X(t))_{t \in[0, T]}$, and consider a sequence of process which can be inductively defined by

$$
X_{0}^{N}(t=0)=X_{0}^{N}, \quad X_{1}^{N}(t)=X_{0}^{N}+\sum_{\ell=1}^{k} \frac{h_{\ell}}{N} P_{\ell}\left(\int_{0}^{t \wedge \tau_{1}} \tilde{\lambda}_{\ell}\left(s, N X_{0}^{N}(s)\right) d s\right),
$$

where $\tau_{1}$ is the first time of jump of the process and in general for $n=2, \ldots$, define the following sequence "from one jump to the next"

$$
X_{n}^{N}(t)=X_{0}^{N}+\sum_{\ell=1}^{k} \frac{h_{\ell}}{N} P_{\ell}\left(\int_{0}^{t \wedge \tau_{n}} \tilde{\lambda}_{\ell}\left(s, N X_{n-1}^{N}(s)\right) d s\right)
$$

for any $t \in[0, T]$, where the sequence $\left(\tau_{n}\right)_{n \geq 0}$ define the time of the $n^{\text {th }}$ jump of the process and given by

$$
\tau_{n}=\inf \left\{t \geq 0, \sum_{\ell=1}^{k} P_{\ell}\left(\int_{0}^{t} \tilde{\lambda}_{\ell}(s, X(s)) d s\right)=n\right\} .
$$

By an inductive argument and the properties of Poisson processes, we show that each $X_{n}$ is adapted and cadlàg.

By the Jensen's inequality $\left(a_{1}+a_{2}+\ldots .+a_{m}\right)^{p} \leq m^{p-1}\left(a_{1}^{p}+a_{2}^{p}+\ldots+a_{m}^{p}\right), a_{i} \geq 0, \forall i=1,2, \ldots, m$ and $p \geq 2$, we have the following inequality

$$
\begin{aligned}
\left|X_{n}^{N}(t)\right|^{p} & \leq(k+2)^{p-1}\left(\left|X_{0}^{N}\right|^{p}+\sum_{\ell=1}^{k}\left|\frac{h_{\ell}}{N} Q_{\ell}\left(\int_{0}^{t \wedge \tau_{n}} \tilde{\lambda}_{\ell}\left(s, N X_{n-1}^{N}(s)\right) d s\right)\right|^{p}\right. \\
& \left.+\left|\int_{0}^{t \wedge \tau_{n}} \frac{1}{N} F\left(s, N X_{n-1}^{N}(s)\right) d s\right|^{p}\right) .
\end{aligned}
$$

By Eq.(16), the stopped stochastic sequence $\left(X_{n}^{N}(t)\right)_{t \in[0, T]}$ remain constant; i.e $X_{n}^{N}(t)=X_{n-1}^{N}(t)$ on $\left[0, \tau_{n+1}\right.$ [ by construction and it is easy to show that $\left(\tau_{n}\right)_{n \geq 0}$ is an increasing sequence which grow to infinity as $n \rightarrow \infty$. Then the following inequality holds 


$$
\begin{aligned}
\mathbb{E}\left[\sup _{0 \leq r \leq t \wedge \beta_{K}(X)}\left|X_{n}^{N}(r)\right|^{p}\right] & \leq(k+2)^{p-1} \mathbb{E}\left\{\left|X_{0}^{N}\right|^{p}+\sup _{0 \leq r \leq t \wedge \beta_{K}(X)}\left|\int_{0}^{r \wedge \tau_{n}} \frac{1}{N} F\left(s, N X_{n-1}^{N}(s)\right) d s\right|^{p}\right. \\
& \left.+\left\|h_{\ell}\right\|_{\infty} \sum_{\ell=1}^{k} \sup _{0 \leq r \leq t \wedge \beta_{K}(X)}\left|\frac{1}{N} Q_{\ell}\left(\int_{0}^{r \wedge \tau_{n}} \tilde{\lambda}_{\ell}\left(s, N X_{n-1}^{N}(s)\right) d s\right)\right|^{p}\right\}
\end{aligned}
$$

where $\|\cdot\|_{\infty}$ is the infinit norm. By using Holderś inequality and the martingale inequality, together with condition (13), (14), we obtain for $1<p<\infty$

$$
\begin{aligned}
& \mathbb{E}\left(\sup _{0 \leq r \leq t \wedge \beta_{K}(X)} \mid \int_{0}^{r \wedge \tau_{n}} \frac{1}{N} F\left(s,\left.N X_{n-1}^{N}(s) d s\right|^{p} \leq\right.\right. 3^{p-1}\left\{\mathbb{E}\left(\frac{1}{N} \int_{0}^{t \wedge \tau_{n}}|F(s, 0)| d s\right)^{p}\right. \\
&+\mathbb{E}\left(\sup _{0 \leq r \leq t \wedge \beta_{K}(X)}\left|X_{n-1}^{N}(s)\right|^{p}\right) \\
&\left.\mathbb{E}\left(\int_{0}^{t \wedge \tau_{n}} L(s) d s\right)^{p}\right\} \\
&< \infty . \\
& \mathbb{E}\left(\sup _{0 \leq r \leq t \wedge \beta_{K}(X)}\left|\tilde{Q}_{\ell}^{N}\left(r \wedge \tau_{n}\right)\right|^{p}\right) \leq C(p) \mathbb{E}\left(\left|\tilde{Q}_{\ell}^{N}\left(t \wedge \tau_{n}\right)\right|^{p}\right) \text { for some } p \geq 2,
\end{aligned}
$$

where $C(p)=\left(\frac{p}{p-1}\right)^{p}$ is a positive constant. Substituting the above inegalities in (19), we have the result

$$
\mathbb{E}\left[\sup _{0 \leq r \leq t \wedge \beta_{K}(X)}\left|X_{n}(r)\right|^{p}\right]<\infty .
$$

Uniqueness holds by setting $X^{N}(t)=\lim _{n \rightarrow \infty} X_{n}^{N}(t)$ and using Gronwall inequality. If $\left(X_{n}^{N}(t)\right)_{t \in[0, T]}$ and $\left(Y_{n}^{N}(t)\right)_{t \in[0, T]}$ are two solutions for some $T>0$, and

$$
h_{n}(t)=\mathbb{E}\left(\sup _{0 \leq r \leq t \wedge \beta_{K}(X)}\left|X_{n}^{N}(r)-Y_{n}^{N}(r)\right|^{2}\right),
$$

we obtain from inequality (19) for the case $p=2$,

$$
h_{n}(t) \leq A \mathbb{E} \exp \left(2 \int_{0}^{t \wedge \tau_{n}} L^{2}(r) d r\right),
$$

where $A=2 \mathbb{E}\left|X_{0}^{N}-Y_{0}\right|^{2}$. Taking $X_{0}^{N}=Y_{0}^{N}$ the same initial condition of the processes, we get $h_{n}(t) \equiv 0$, this lead to with probability one $X(t)=Y(t)$ is the unique solution to Eq.(6) for all $t>0$.

Lemme 2.4 Suppose that condition (14) hold and

$$
|F(t, x)-F(t, y)| \leq L_{n}(t)|x-y|,
$$

for all $t \in[0, T]$ with $|x| \vee|y| \leq n$, where $L_{n}(t) \in L^{1}\left([0, T] ; \mathbb{R}_{+}\right)$. Then Eq.(6) has a unique maximal local solution $X(t)$ for $t \in\left[0, \beta_{K}(X)\right]$. Moreover, $X(t)$ explodes at time $\beta_{K}(X)$ if $\beta_{K}(X)<T$. Otherwise, the solution $X(t)$ exists in $[0, T]$. 
Proof This result can be proved by the standard truncation procedure. For each $k \geq 1$, define the troncature function;

$$
\tilde{\lambda}_{\ell, k}(t, x)=\tilde{\lambda}_{\ell}\left(t, \frac{|x| \wedge k}{|x|} x\right)
$$

where we set $\frac{|x| \wedge k}{|x|} x=0$ when $x=0$. By Lemma 2.3, there exists a unique solution to the following equation

$$
X_{n}^{N}(t)=X_{0}^{N}+\sum_{\ell=1}^{k} h_{\ell} P_{\ell}\left(\int_{0}^{t \wedge \tau_{n}} \tilde{\lambda}_{\ell}\left(r, X_{n-1}^{N}(r)\right) d r\right) \mathrm{t} \in[0, T]
$$

with the initial value $X_{0}^{N}$. Define the stopping time

$$
\beta_{J}=\inf \left\{t \geq 0,\left|X_{n}^{N}(t)\right| \geq J\right\} .
$$

We can observe that for $t \in\left[0, \beta_{J}\right]$

$$
\tilde{\lambda}_{J}\left(t, X_{J}^{N}(t)\right)=\tilde{\lambda}_{J+1}\left(t, X_{J}^{N}(t)\right)
$$

This leads to

$$
X_{J}^{N}(t)=X_{j+1}^{N}(t), \mathrm{t} \in\left[0, \beta_{J}\right] .
$$

Hence $\left(\beta_{J}\right)_{J \geq 0}$ is an increasing sequence. Set $\beta_{K}(X)=\lim _{J \rightarrow \infty} \beta_{J}$ which may be eventually infinite. For $t \in\left[0, \beta_{K}(X)\right]$, define $X^{N}(t)=\lim _{J \rightarrow \infty} X_{J}^{N}(t), t \in\left[0, \beta_{J}\right]$. We obtain that $\left(X^{N}(t)\right)_{t \geq 0}$ is the unique maximal local solution to Eq.(6) for $t \in\left[0, \beta_{K}(X)\right]$. The solution is global if $\beta_{K}(X)=\infty$.

To study the moment estimate of the process $\left(X^{N}(t)\right)_{t \geq 0}$ by Itô's formula, we need first the following result.

Proposition 2.5 The stochastic process $\left(X^{N}(t)\right)_{t \geq 0}$ is continuous in probability, i.e for all $s, t$ and a positive real number $b$,

$$
\lim _{s \rightarrow t} \mathbb{P}\left(\left|X^{N}(t)-X^{N}(s)\right|>b\right)=0 .
$$

Hence $\left(X^{N}(t)\right)_{t \geq 0}$ has a cadlàg modification $\left(Z^{N}(t)\right)_{t \geq 0}$ and the stochastic process $\left(Z^{N}\left(t^{-}\right)\right)_{t \geq 0}$ where $Z^{N}\left(t^{-}\right)=\lim _{s \rightarrow t^{-}} Z^{N}(s)$ is a continuous process which can be define as follows.

If $Q$ is a standard Poisson random measures on $\mathbb{R}_{+}^{2}$ (i.e. with mean measure Lebesgue), then

$$
\int_{0}^{t} \int_{0}^{\infty} \mathbf{1}_{\{u \leq \lambda(s)\}} Q(d s, d u) \text { is a rate } \lambda(t) \text { Poisson process. }
$$

Then the stochastic process $\left(X^{N}(t)\right)_{t \geq 0}$ has the same distribution with the stochastic process $\left(Z^{N}(t)\right)_{t \geq 0}$, solution of the following equation:

$$
\tilde{Z}(t)=\tilde{Z}(0)+\sum_{\ell=1}^{k} \frac{h_{\ell}}{N} \int_{0}^{t} \int_{0}^{\infty} \mathbf{1}_{\left.\left\{u \leq \tilde{\lambda}_{\ell}\left(s, N \tilde{Z}\left(s^{-}\right)\right)\right)\right\}} Q(d s, d u) .
$$

where $\tilde{Z}(t)=\frac{1}{N} Z(t)$

Remark 2.6 We would assume more generally for the periodic stochastic intensity $\tilde{\lambda}_{\ell}\left(s, N X^{N}(s)\right)$ that:

$$
\tilde{\lambda}_{\ell}\left(s, N X^{N}(s)\right)=N \tilde{\tilde{\lambda}}_{\ell}\left(s, X^{N}(s)\right) \text { and } \frac{1}{N} F\left(s, N X^{N}(s)\right) \rightarrow b(s, x(s)) .
$$

as $N \rightarrow \infty$. 
Remark 2.7 Define by $(\tilde{Z}(t))_{t \geq 0}$ the solution to Eq.(21), and $\Delta \tilde{Z}(t)=\tilde{Z}(t)-\tilde{Z}(t)$ the jump process where $\tilde{Z}\left(t^{-}\right)=\lim _{s \rightarrow t^{-}} \tilde{Z}(s)$. The stochastic process $\Delta \tilde{Z}(t) \in\left\{0, \frac{1}{N}, \frac{-1}{N}\right\}$. As $N \rightarrow \infty, \Delta \tilde{Z}(t) \equiv 0$, the process $(\tilde{Z}(t))_{t \geq 0}$ has a continuous limits and the stochastic process $\left(X^{N}(t)\right)_{t \geq 0}$ is indistinguishable from $(\tilde{Z}(t))_{t \geq 0}$ on $[0, T]$. Then the following result gives more details on the mode of convergence.

Proposition 2.8: Law of Lagre Numbers, (Britton, T. and Pardoux, E., 2019) Assume that the assumption $\left(H_{1}\right)$ and $\left(H_{2}\right)$ hold, that $b(t, x(t))=\sum_{\ell=1}^{k} h_{\ell} \lambda_{\ell}(t, x(t))$ is locally Lipschitz as a function of $x$, locally uniformly in $t$ that is $L(t)=C($ a constant) in Lemma 2.3. Let $\left(X^{N}(t)\right)_{t \geq 0}$ denote the solution to Eq.(6). Then $\lim _{N \rightarrow \infty} X^{N}(t)=x(t)$ a.s locally uniformly in $t$, where $x(t)$ is solution to ODE Eq.(5).

Corollary 2.9 Assume that the assumption of proposition (2.8) are satisfied and $\sum_{\ell=1}^{k} h_{\ell} \lambda_{\ell}(t+\tau, x(t))=\sum_{\ell=1}^{k} h_{\ell} \lambda_{\ell}(t, x(t))$ for some $\tau>0$.

(i) If $x(t)$ is solution to Eq.(5), then $x(t+\tau)$ is also solution to Eq.(5).

(ii) The ODE Eq.(5) has a $\tau$-periodic solution for all initial condition $x(0)=x_{0}$.

Note if $\tilde{\lambda}_{\ell}(t+\tau, X(t))=\tilde{\lambda}_{\ell}(t, X(t))$, for some $\tau>0, \forall t \in[0, T]$ then $F\left(t+\tau, X^{N}(t)\right)=F\left(t, X^{N}(t)\right)$ and so for each integer $m, b(t+m \tau, x(t))=b(t, x(t))$ and corollary 2.9 can be stated in terms of $m \tau$-periodic solutions.

Proof To prove (i), we let $q(t)=x(t+\tau)$ so that $q^{\prime}(t)=b(t+\tau, x(t+\tau))=\lim _{N \rightarrow \infty} \frac{1}{N} F\left(t+\tau, N X^{N}(t)\right)=b(t, q(t))$. To prove $(i i)$, we consider an other solution $q^{1}(t)=$ $x^{1}(t+\tau)$ with initial condition $q^{1}(0)=x_{0}$. By uniqueness of $x_{0}$, we obtain uniqueness of $q(t)$ and the proof is completed.

Remark 2.10 Skorokhod's Representation theorem (Billingsley, P., 1999, See p. 70) proves that there exists a probability space $(\Omega, \mathcal{F}, \mathbb{P})$ such that $\lim _{N \rightarrow \infty} \tilde{Z}(t)=x(t), \mathbb{P}$-almost surely.

Let's define the compensated random measure $\tilde{Q}(d s, d u)=Q(d s, d u)-\lambda(d u) d t$. Then, the process $(Z \tilde{(} t))_{t \geq 0}$ solution of the Eq.(21) is differentiable and as the form written as follows

$$
d \tilde{Z}(t)=\frac{1}{N} F\left(t, N \tilde{Z}\left(t^{-}\right)\right) d t+\sum_{\ell=1}^{k} h_{\ell} \lambda_{\ell}(t+\tau, x(t))_{\ell=1}^{k} \frac{h_{\ell}}{N} \int_{0}^{\infty} \mathbf{1}_{\left\{u \leq \tilde{\lambda}_{\ell}\left(t, N \tilde{Z}\left(t^{-}\right)\right)\right\}} \tilde{Q}_{\ell}(d t, d u) .
$$

Let $C^{1,2}\left(\mathbb{R} \times \mathbb{R}^{n} ; \mathbb{R}\right)$, denote the family of all functions $V(t, z)$ on $\mathbb{R} \times \mathbb{R}^{n}$, which are twice continuously differentiable in $z$ and once in $t$. For each $V(t, z) \in C^{1,2}\left(\mathbb{R} \times \mathbb{R}^{n} ; \mathbb{R}\right)$, we define an Itô operator $\mathcal{L} V(t, z)$ of the function $V(t, z)$ associated with the Eq.(23) of (SDEsGp) by

$$
\begin{aligned}
\mathcal{L} V(t, \tilde{Z}) & =V_{t}(t, \tilde{Z})+V_{x}(t, \tilde{Z}) \\
& +\sum_{\ell=1}^{k} \frac{h_{\ell}}{N} \int_{0}^{\infty}\left[V\left(t, \tilde{Z}+\mathbf{1}_{\left\{u \leq \tilde{\lambda}_{\ell}\left(t, N \tilde{Z}\left(t^{-}\right)\right)\right\}}\right)-V(t, \tilde{Z})\right. \\
& \left.-V_{z}(t, \tilde{Z}) \mathbf{1}_{u \leq \tilde{\lambda}_{\ell}\left(t, N \tilde{Z}\left(t^{-}\right)\right)}\right] \lambda(d u) .
\end{aligned}
$$

where

$$
V_{t}=\frac{\partial V}{\partial t}, \quad V_{z}=\left(\frac{\partial V}{\partial z_{1}}, \frac{\partial V}{\partial z_{2}}, \ldots ., \frac{\partial V}{\partial z_{d}}\right)
$$


The Itô formula for (SDEsGp) type stochastic integrals is

$$
\begin{aligned}
V\left(t, \tilde{Z}\left(t^{-}\right)\right)-V(0, \tilde{Z}(0)) & =\int_{\ell=1}^{k} h_{\ell} \lambda_{\ell}(t+\tau, x(t))_{0}^{t} \mathcal{L} V\left(s, \tilde{Z}\left(s^{-}\right)\right) d s+\int_{0}^{t} V_{z}\left(s, \tilde{Z}\left(s^{-}\right)\right) d s \\
& +\sum_{\ell=1}^{k} \frac{h_{\ell}}{N} \int_{0}^{t} \int_{0}^{\infty}\left\{V\left(s, \tilde{Z}\left(s^{-}\right)+\mathbf{1}_{\left\{u \leq \tilde{\lambda}_{\ell}\left(s, N \tilde{Z}\left(s^{-}\right)\right)\right\}}\right)\right. \\
& \left.-V\left(s, \tilde{Z}\left(s^{-}\right)\right)\right\} \tilde{Q}_{\ell}(d s, d u) .
\end{aligned}
$$

Lemme 2.11 Let's Lemma 2.3 and Lemma 2.4 hold. Suppose that there are two functions $V \in C^{1,2}\left(\left[0, \infty\left[\times \mathbb{R}^{n} ; \mathbb{R}_{+}\right)\right.\right.$, $p \in L^{1}\left([0, T] ; \mathbb{R}_{+}\right)$and $q \in L^{1}\left([0, T] ; \mathbb{R}_{+}\right)$such that

$$
\begin{gathered}
\lim _{|z| \rightarrow \infty}\left[\inf _{0 \leq t \leq T} V(t, z)\right]=\infty . \\
\mathcal{L} V\left(t, \tilde{Z}\left(t^{-}\right)\right) \leq-p(t) V\left(t, \tilde{Z}\left(t^{-}\right)\right)+q(t) .
\end{gathered}
$$

Then Eq.(23) has a unique solution $(\tilde{Z}(t))_{t \geq 0}$, and the solution satisfies the following moment estimate

$$
\begin{aligned}
\mathbb{E}\left(V\left(t, \tilde{Z}\left(t^{-}\right)\right)\right) & \leq \exp \left(-\int_{0}^{t} p(s) d s\right) \mathbb{E}(V(0, \tilde{Z}(0))) \\
& +\int_{0}^{t} q(s) \exp \left(-\int_{s}^{t} p(u) d u\right) d s .
\end{aligned}
$$

Proof Assumption (24) gives nonexplosion condition for solution to a more general stochastic differential equation by Lyapunov functions method. The proof is motivated by (Zhang, X. and all, 2015). From Lemma 2.4, we obtain there is a unique maximal local solution $(\tilde{Z}(t))_{t \geq 0}$ on $\left[0, \beta_{K}(Z)\right]$, where $\tilde{\beta}_{K}(Z)$ is the explosion time of the process $(\tilde{Z}(t))_{t \geq 0}$. We need to prove that $\tilde{\beta}_{K}(Z)=T$ and will proceed by absurd. If it is not, there exist two constants $\varepsilon$ and $S<T$ such that

$$
\mathbb{P}\left(\left\{\tilde{\beta}_{K}(Z) \leq S\right\}\right)>\varepsilon .
$$

Thus we can find a sufficiently large integer $k_{0}$ such that

$$
\mathbb{P}\left(\left\{\tilde{\beta}_{k} \leq S\right\}\right)>\varepsilon, \forall k \geq k_{0},
$$

where $\tilde{\beta}_{k}=\inf \left\{t \in[0, T],\left|Z_{k}(t)\right| \geq k\right\}$. By Itô's formula and inequality 25 given by Lemma 2.11 , we have the following results for any $t \in[0, S]$

$$
\begin{aligned}
\mathbb{E}\left[V\left(t \wedge \tilde{\beta}_{k}, \tilde{Z}\left(t^{-} \wedge \tilde{\beta}_{k}\right)\right)\right] & =V(0, \tilde{Z}(0))+\mathbb{E}\left\{\int_{0}^{t \wedge \tilde{\beta}_{k}} \mathcal{L}(V(s, \tilde{Z}(s))) d s\right\} \\
& \leq V(0, \tilde{Z}(0))+\mathbb{E}\left(\int_{0}^{t \wedge \tilde{\beta}_{k}} q(s) d s\right)-\mathbb{E}\left(\int_{0}^{t \wedge \tilde{\beta}_{k}} p(s) V(s, \tilde{Z}(s))\right) d s .
\end{aligned}
$$


Thus we get

$$
\mathbb{E}\left[V\left(S \wedge \tilde{\beta}_{k}, \tilde{Z}\left(S^{-} \wedge \tilde{\beta}_{k}\right)\right)\right]<\infty .
$$

Also,

$$
\mathbb{E}\left[\mathbf{1}_{\left\{\tilde{\beta}_{k} \leq S^{-}\right\}} V\left(\tilde{\beta}_{k}, \tilde{Z}\left(\tilde{\beta}_{k}\right)\right)\right]<\infty .
$$

Define by

$$
\eta(k)=\inf \left\{V(t, z) ;(t, z) \in[0, T] \times \mathbb{R}^{d},|z| \geq k\right\} .
$$

Then by condition (24) of Lemma 2.11, we get $\lim _{k \rightarrow \infty} \eta(k)=\infty$. From inequality (27) and inequality (29), it follows the next contradiction

$$
\varepsilon \eta(k)<\eta(k) \mathbb{P}\left(\left\{\tilde{\beta}_{k} \leq S\right\}\right)<\infty, \forall S<T .
$$

Therefore, the stochastic process $\left(Z^{N}(t)\right)_{t \geq 0}$ is the unique bounded solution to Eq.(23) on [0,T].

Now we prove the moment estimate. By the integration formula by part or more generally by Itô's formula applaying to the process $\exp \left(\int_{0}^{t} p(u) d u\right) V\left(t, \tilde{Z}\left(t^{-}\right)\right)$, it follows that for any $0 \leq t \leq T$

$$
\begin{aligned}
\exp \left(\int_{0}^{t} p(u) d u\right) V\left(t, \tilde{Z}\left(t^{-}\right)\right) & =V(0, \tilde{Z}(0))+\int_{0}^{t} p(s) \exp \left(\int_{0}^{s} p(u) d u\right) V\left(s, \tilde{Z}\left(s^{-}\right)\right) d s \\
& +\int_{0}^{t} \exp \left(\int_{0}^{s} p(u) d u\right) d V(s, \tilde{Z}(s)) \\
& +\left\langle\exp \left(\int_{0}^{\infty} p(u) d u\right), V(., \tilde{Z}(.))\right\rangle_{t^{-}}
\end{aligned}
$$

where $\langle., .\rangle_{t}$ is the quadratic variation. Taking expectation, we get

$$
\begin{aligned}
\mathbb{E}\left(\exp \left(\int_{0}^{t} p(u) d u\right) V\left(t, \tilde{Z}\left(t^{-}\right)\right)\right) & =\mathbb{E}(V(0, \tilde{Z}(0))) \\
& +\mathbb{E}\left(\int_{0}^{t} p(s) \exp \left(\int_{0}^{s} p(u) d u\right) V\left(s, \tilde{Z}\left(s^{-}\right)\right) d s\right) \\
& +\mathbb{E}\left(\int_{0}^{t} \exp \left(\int_{0}^{s} p(u) d u\right) d V(s, \tilde{Z}(s))\right), \\
\leq & \mathbb{E}(V(0, \tilde{Z}(0))) \\
& +\mathbb{E}\left(\int_{0}^{t} p(s) \exp \left(\int_{0}^{s} p(u) d u\right) V\left(s, \tilde{Z}\left(s^{-}\right)\right) d s\right) \\
& +\int_{0}^{t} \exp \left(\int_{0}^{s} p(u) d u\right)\left[-p(s) V\left(s, \tilde{Z}\left(s^{-}\right)\right)+q(s)\right] d s, \\
\mathbb{E}\left(\exp \left(\int_{0}^{t} p(u) d u\right) V\left(t, \tilde{Z}\left(t^{-}\right)\right)\right) & \leq \mathbb{E}(V(0, \tilde{Z}(0)))+\int_{0}^{t} q(s) \exp \left(\int_{0}^{s} p(u) d u\right) d s,
\end{aligned}
$$


which implies

$$
\mathbb{E}\left(V\left(t, \tilde{Z}\left(t^{-}\right)\right)\right) \leq \exp \left(-\int_{0}^{t} p(u) d u\right) \mathbb{E}(V(0, \tilde{Z}(0)))+\int_{0}^{t} q(s) \exp \left(-\int_{s}^{t} p(u) d u\right) d s,
$$

for every time $t \in[0, T]$.

\subsection{Periodic Solution of Stochastic Differential Equations Driven by General Counting Processes (SDEsGp)}

Periodic mouvement is very universal in nature, for exemple climate changes every three or four seasons. So for periodic stochastic intensity, an importante aspect is to ensure uniqueness of a periodic solution to Eq.(6).

In this paragraph, we discus the periodicity of the solution of Eq.(6). First, we recall some definitions of periodic Markov processes which are found in ( $\mathrm{Li}, \mathrm{D}$ and $\mathrm{Xu}, \mathrm{D}, 2013)$.

Definition 2.12 A stochastic process $z(t, \varpi)$ with values in Banach space $\mathbb{R}^{n}$, defined for $t \geq 0$ on a probability space $\left(\Omega, \mathcal{F},\left\{\mathcal{F}_{t}\right\}, \mathbb{P}\right)$, is called a Markov process if, for all $A \in \mathcal{B}, 0 \leq v \leq t$,

$$
\mathbb{P}\left(\left\{z(t, \varpi) \in A \mid \mathcal{F}_{v}\right\}\right)=\mathbb{P}(\{z(t, \varpi) \in A \mid z(v, \varpi)\}),
$$

where $\mathcal{F}_{v}$ is the $\sigma$-algebra of events generated by all events of the form $\{z(u, \varpi) \in A, u \leq v\}$ and $\mathcal{B}$ denotes the $\sigma$-algebra of Borel set in $\mathbb{R}^{n}$

Definition 2.13 A stochastic process $z(t, \varpi)$ is said to be periodic with period $\tau>0$ if its finite dimensional distributions are periodic with period $\tau>0$, i.e., for any positive integer $m$ and any moments of time $t_{1}, \ldots, t_{m}$, the joint distributions of the random variables $z\left(t_{1}+k \tau, \varpi\right), \ldots ., z\left(t_{m}+k \tau, \varpi\right)$ are independent of $k(k= \pm 1, \pm 2, \ldots$.$) .$

The transition function of a Markov process, $p(v, z(v), t, A)=\mathbb{P}(z(t) \in A \mid z(v))$, a.s, is called periodic if $t \mapsto p(v, z(v), t+v, A)$ is periodic in $v$.

Later on we shall often denote a family of Markov processes by $z_{t}^{\left(t_{0}, \phi\right)}(\varpi)$ for all $t_{0} \in \mathbb{R}_{+}$and $z_{t_{0}}=z\left(t_{0}+s\right)=\phi(s)$ a bounded $\mathcal{F}_{0}$-mesurable function.

Definition 2.14 The Markov families $z_{t}^{\left(t_{0}, \phi\right)}(\varpi)$ are said to be uniformly bounded, if for each $\alpha>0, t_{0} \in \mathbb{R}_{+}$, there exists a positive constant $\theta=\theta(\alpha)$ which is independent of $t_{0}$ such that $\mathbb{E}\left(\left|z\left(t_{0}\right)\right|\right) \leq \alpha$ implies that $\mathbb{E}\left(\mid z\left(t, t_{0}, z\left(t_{0}\right) \mid\right) \leq \theta, t \geq t_{0}\right.$. In a general way, the Markov families $z_{t}^{\left(t_{0}, \phi\right)}(\varpi)$ are said to be $p$-uniformly bounded if $\mathbb{E}(|\cdot|)$ is replaced by $\mathbb{E}\left(||^{p}\right)$.

Theorem 2.15 Suppose that for any $T \in[0, \infty[$, Lemma 2.4 and Lemma 2.11 hold. If there exits a positive constant $C$ such that :

$$
\int_{0}^{t} q(s) \exp \left(-\int_{s}^{t} p(u) d u\right) d s<C, \forall \mathrm{t} \in[0, \infty[,
$$

and there are two positive constants $\alpha$ and $\gamma$ such that for all $t \in[0, \infty[$,

$$
\alpha|z|^{p} \leq V(t, z) \leq \gamma|z|^{p}
$$

Then the solution of Eq.(23) is p-uniformely bounded and Eq.(23) admits an $\tau$-periodic stochastic solution.

Proof For any $T \in\left[0, \infty\left[\right.\right.$, since Lemma 2.3, Lemma 2.4 and Lemma 2.11 hold for the stochastic process $(\tilde{Z}(t))_{t \geq 0}$ solution for Eq.(23), then Eq.(23) has a unique solution $\tilde{Z}(t)$ on $[0, \infty[$ and this solution satisfies the following inequality

$$
\mathbb{E}\left(V\left(t, \tilde{Z}\left(t^{-}\right)\right)\right) \leq \exp \left(-\int_{0}^{t} p(u) d u\right) \mathbb{E}(V(0, \tilde{Z}(0)))+\int_{0}^{t} q(s) \exp \left(-\int_{s}^{t} p(u) d u\right) d s,
$$

$\forall t \in\left[0, \infty\left[\right.\right.$. With the conditions (31) and (32), we get the $p$-uniformly bounded of the solution. To fit together with assumption $\left(\mathrm{H}_{1}\right)$ the transition probabilities function to Eq.(23) is $\tau$-periodic ( $\mathrm{Li}, \mathrm{D}$ and $\mathrm{Xu}, \mathrm{D}, 2013$ ), this yield there exits an $\tau$-periodic solution to Eq.(23). This complete the proof.

Our last point is to give sufficient conditions for uniqueness of the periodic stochastic solution. We need first the following definitions.

Definition 2.16 The $\tau$-periodic stochastic solution $\tilde{Z}^{*}(t)$ of Eq.(23) is said to be stable in $p$-th moment if for arbitrarily given $\varepsilon>0$, there exists a $\delta=\delta(\varepsilon)$ such that $\mathbb{E}\left(\left|Z(0)-\tilde{Z}^{*}(0)\right|^{p}\right)<\delta$ implies that: 


$$
\mathbb{E}\left(\left|\tilde{Z}(t)-\tilde{Z^{*}}(t)\right|^{p}\right)<\varepsilon, \quad \mathrm{t} \geq 0,
$$

where $(Z(t))_{t \geq 0}$ is an arbitraly solution of Eq.(23) whith initial condition $\mathbb{E}\left(|\tilde{Z}(0)|^{p}\right)<\infty$.

Definition 2.16 The $\tau$-periodic stochastic solution $\tilde{Z}^{*}(t)$ to Eq.(23) is said to be asymptotically stable in $p$-th moment, if it is stable in $p$-th moment and satifies:

$$
\lim _{t \rightarrow \infty} \mathbb{E}\left(\left|\tilde{Z}(t)-\tilde{Z^{*}}(t)\right|^{p}\right)=0 .
$$

We are going now to give sufficients condition on the asymptotic stability of solution to Eq.(23) which ensure the uniqueness of stochastic periodic solution. Let $\tilde{Z}^{z_{1}}(t)$ and $\tilde{Z}^{z_{2}}(t)$ be two periodic solutions to Eq.(23) with initial values $z_{1}$ and $z_{2}$ respectively. Then we have:

$$
\begin{aligned}
\tilde{Z}^{z_{1}}(t)-\tilde{Z}^{z_{2}}(t) & =z_{1}-z_{2}+\int_{0}^{t} \frac{1}{N}\left[F\left(s, N \tilde{Z}^{z_{1}}\left(s^{-}\right)\right)-F\left(s, N \tilde{Z}^{z_{2}}\left(s^{-}\right)\right)\right] d s \\
& +\sum_{\ell=1}^{k} \frac{h_{\ell}}{N} \int_{0}^{t} \int_{0}^{\infty} \mathbf{1}_{\left\{u \leq \tilde{\lambda}_{\ell}\left(s, N \tilde{Z}^{z_{1}}\left(s^{-}\right)\right)\right\} \backslash\left\{u \leq \tilde{\lambda}_{\ell}\left(s, N \tilde{Z}^{z_{2}}\left(s^{-}\right)\right)\right\}} \tilde{Q}_{\ell}(d s, d u) .
\end{aligned}
$$

Let's define $W(t, v, z)$ a given function $W \in C^{1,2}\left(\left[0, \infty\left[\times \mathbb{R}^{n} ; \mathbb{R}_{+}\right)\right.\right.$. An operator $\mathcal{L} W:\left[0, \infty\left[\times \mathbb{R}^{n} \times \mathbb{R}^{n} \rightarrow \mathbb{R}\right.\right.$ associated with Eq.(34) is defined as following:

$$
\begin{aligned}
\mathcal{L} W(t, v, z) & =W_{t}(t, v-z)+W_{v}(t, v-z)[F(t, v)-F(t, z)] \\
& +\int_{0}^{\infty}\left[W\left(t, v-z+\mathbf{1}_{\left.\left\{u \leq \tilde{\lambda}_{\ell}(t, v)\right)\right\} \backslash\left\{u \leq \tilde{\lambda}_{\ell}(t, z)\right\}}\right)-W(t, v-z)\right. \\
& \left.-W_{v}(t, v-z) \mathbf{1}_{\left.\left\{u \leq \tilde{\lambda}_{\ell}(t, v)\right)\right\} \backslash\left\{u \leq \tilde{\lambda}_{\ell}(t, z)\right\}}\right] \lambda(d u) .
\end{aligned}
$$

Theorem 2.17 Assume that all inequalities in theorem hold and there exists a function $W \in C^{1,2}\left(\left[0, \infty\left[\times \mathbb{R}^{n} ; \mathbb{R}_{+}\right)\right.\right.$such that:

$$
\theta|z|^{p} \leq W(t, z) \leq \vartheta|z|^{p}
$$

and

$$
\mathcal{L} W(t, v, z) \leq \alpha(t) W(t, v-z),
$$

where $\theta, \vartheta$ are positive constants and $\alpha(t)$ has the property:

$$
\lim _{t \rightarrow \infty} \exp \left(-\int_{0}^{t} \alpha(s) d s\right)=0
$$

Then the $\tau$-periodic stochastic solution $\tilde{Z^{*}}(t)$ of Eq.(23) is asymptotically stable in $p$-th moment. Therefore Eq.(23) has a unique stochastic periodic solution.

Remark 2.18 Condition (37) hold for $\alpha(s)=C$ (constant), $\forall s \in[0, t]$ for all $t \geq 0$ and more generally all polynomial functions.

Proof Applying Itô's formula to the process exp $\left(\int_{0}^{t} \alpha(s) d s\right) W\left(t, \tilde{Z}(t)-\tilde{Z}^{*}(t)\right)$ and taking the expectation, it yields 


$$
\begin{aligned}
\mathbb{E}\left[\exp \left(\int_{0}^{t} \alpha(s) d s\right) W\left(t, \tilde{Z}(t)-\tilde{Z}^{*}(t)\right)\right] & =\mathbb{E}\left[W\left(0, \tilde{Z}(0)-\tilde{Z}^{*}(0)\right)\right] \\
& +\mathbb{E}\left[\int_{0}^{t} \alpha(s) \exp \left(\int_{0}^{s} \alpha(u) d u\right) W\left(s, \tilde{Z}(s)-\tilde{Z}^{*}(s)\right) d s\right] \\
& +\mathbb{E}\left[\int_{0}^{t} \exp \left(\int_{0}^{s} \alpha(u) d u\right) \mathcal{L} W\left(s, \tilde{Z}(s), \tilde{Z^{*}}(s)\right) d s\right]
\end{aligned}
$$

Using (36) and (37), we get

$$
\theta \exp \left(\int_{0}^{t} \alpha(s) d s\right) \mathbb{E}\left(\left|\tilde{Z}(t)-\tilde{Z}^{*}(t)\right|^{p}\right) \leq \mathbb{E}\left[W\left(0, \tilde{Z}(0)-\tilde{Z}^{*}(0)\right)\right] \leq \vartheta \mathbb{E}\left(\left|\tilde{Z}(0)-\tilde{Z}^{*}(0)\right|^{p}\right) .
$$

Then,

$$
\mathbb{E}\left(\left|\tilde{Z}(t)-\tilde{Z}^{*}(t)\right|^{p}\right) \leq \frac{\vartheta}{\theta} \exp \left(-\int_{0}^{t} \alpha(s) d s\right) \mathbb{E}\left(\left|\tilde{Z}(0)-\tilde{Z}^{*}(0)\right|^{p}\right),
$$

which prove that the $\tau$-periodic stochastic solution $\tilde{Z}^{*}(t)$ to Eq.(23) is stable in $p$-th moment for $\alpha(t) \geq 0$. Next by condition (37) and taking the limit, we get $\lim _{t \rightarrow \infty} \mathbb{E}\left(\left|\tilde{Z}(t)-\tilde{Z}^{*}(t)\right|^{p}\right)=0$. Then, the $\tau$-periodic stochastic solution $\tilde{Z}^{*}(t)$ is asymptotically stable in $p$-th moment. Therefore, the $\tau$-periodic stochastic solution is unique. This complete the proof.

\section{Application to Malaria Under Periodic Environment}

\subsection{Description of the Model}

We considere a mathematical compartmental model to describe a Malaria epidemic under periodic environment and assume that the human total population size $K$ varies little over the time, which comes down to consider it constant in a given region. We denote by $S_{h}(t)$ and $I_{h}(t)$ the total size of susceptible and infectious human population respectively at time $t$. Then to study the dynamics of malaria in human population, we need to know the dynamics of $I_{h}(t)$. For mosquitoes population, we considere two stages of mosquitoes' growth: the juvenile stage which size is denoted by $L_{v}(t)$ and the adult mosquitoes are divided in two compartments. Let $S_{v}(t)$ and $I_{v}(t)$ denote the total size of susceptible and infectious mosquitoes respectively at time $t$. We denote the biting rate of mosquitoes by $\beta_{v}(t)$ which is the number of bites per mosquito per unit time at time $\mathrm{t}$ and depend of environment parameters. Let $p$ and $\ell$ be the probabilities that a mosquito arrives randomly at a human and picks the human if he is infectious and susceptible, respectively. Since infectious humans are more attractive to mosquitoes, we assume that $p \geq l$. Several event happen during the transmission of malaria

i) Obviously, we assume that the probability that an infectious mosquito picks a susceptible human is uniform and given by $\frac{\ell S_{h}(t)}{\ell S_{h}(t)+p I_{h}(t)}$, the ratio between the total bitten susceptible humans over the total bitten humans. Thus, the counting process which count the cumulative occurred infectious humans up to time $t$ is

$$
P_{S I}^{H}\left(\int_{0}^{t} c \beta_{v}(r) \frac{\ell S_{h}(r)}{\ell S_{h}(r)+p I_{h}(r)} I_{v}(r) d r\right),
$$

where $c$ is the probability of transmission of infection from an infectious mosquito to a susceptible human given that the contact between the two occurs, $\beta_{v}(t)$ is a positive, continuous function $\tau$-periodic in time $t$ for some $\tau>0$ and $\left(P_{S I}^{H}(t)\right)_{t \geq 0}$ is a standard Poisson process.

ii) Similary, the probability that a susceptible mosquito picks an infectious human is $\frac{p I_{h}(t)}{\ell S_{h}(t)+p I_{h}(t)}$, the ratio between the total bitten infectious humans over the total bitten humans. The counting process which count the cumulative occurred infectious mosquitoes up to time $t$ is

$$
P_{S I}^{V}\left(\int_{0}^{t} b \beta_{v}(r) \frac{p I_{h}(r)}{\ell S_{h}(r)+p I_{h}(r)} S_{v}(r) d r\right),
$$

where $b$ is the probability of transmission of infection from an infectious human to a susceptible mosquito given that the contact between the two occurs and $\left(P_{S I}^{V}(t)\right)_{t \geq 0}$ is a standard Poisson process. 
iii) After infection, an infectious human can die naturelly or of malaria if the treatment is not effective at constant rates $d_{h}$ and $\delta_{h}$ respectivelly. The counting processes which count the cumulative occurred infectious humans who died up to time $t$ are

$$
P_{I M_{n}}^{H}\left(d_{h} \int_{0}^{t} I_{h}(r) d r\right) \text { and } P_{I M_{m}}^{H}\left(\delta_{h} \int_{0}^{t} I_{h}(r) d r\right)
$$

where $\left(P_{I M_{n}}^{H}(t)\right)_{t \geq 0}$ and $\left(P_{I M_{m}}^{H}(t)\right)_{t \geq 0}$ are standard Poisson processes.

$i v$ ) If the treatment is effective, an infectious human can recover from malaria, this happen at a constant rate $\rho$ and the counting process which count the cumulative occurred recovered infectious humans up to time $t$ is

$$
P_{I G}^{H}\left(\rho \int_{0}^{t} I_{h}(r) d r\right)
$$

where $\left(P_{I G}^{H}(t)\right)_{t \geq 0}$ is a standard Poisson process.

v) After laying eggs on waterfront by adult female mosquitoes, juvenile mosquitoes are born at a varying rate $\lambda_{L}(t)$ which varies according to the water temperature (Paaijmans, K.P. and all, 2009). Thus, the counting process which count the cumulative occurred new born juvenile mosquitoes up to time $t$ is

$$
P_{L N}^{V}\left(\int_{0}^{t} \lambda_{L}(r) N_{v}(r) d r\right)
$$

where $N_{v}(t)=S_{v}(t)+I_{v}(t)$ is the total size of adult female mosquitoes, $\lambda_{L}(t)$ is a positive, continuous function $\tau$-periodic in time t for some $\tau>0$ and $\left(P_{L N}^{V}(t)\right)_{t \geq 0}$ is a standard Poisson process.

vi) Juvenile mosquitoes leave juvenile stage for adulte stage at the varying rate $\lambda_{v}(t)$ which varies according to the air temperature and define the birth rate of adult susceptible mosquitoes (Rubel, F. and all, 2008). The counting process which count the cumulative occurred new born adult susceptible mosquitoes up to time $\mathrm{t}$ is

$$
P_{S N}^{V}\left(\int_{0}^{t} \lambda_{v}(r) L_{v}(r) d r\right)
$$

where $\lambda_{v}(t)$ is a positive, continuous function $\tau$-periodic in time t for some $\tau>0$ and $\left(P_{S N}^{V}(t)\right)_{t \geq 0}$ is a standard Poisson process.

vii) During their development juvenile mosquitoes can die naturally at the varying rate $\mu_{L}(t)$ or for predation at the density-dependent death rate $\alpha$. The counting processes which count the cumulative occurred juvenile mosquitoes who died up to time $t$ are

$$
P_{L_{v} M_{n}}^{V}\left(\int_{0}^{t} \mu_{L}(r) L_{v}(r) d r\right) \text { and } P_{L_{v} M_{p}}^{V}\left(\int_{0}^{t} \alpha L_{v}^{2}(r) d r\right)
$$

where $\mu_{L}(t)$ is a positive, continuous function $\tau$-periodic in time $t$ for some $\tau>0,\left(P_{L_{v} M_{n}}^{V}(t)\right)_{t \geq 0}$ and $\left(P_{L_{v} M_{p}}^{V}(t)\right)_{t \geq 0}$ are standard Poisson processes.

viii) Adult female mosquitoes, susceptible and infectious die naturally at the varying rate $\mu_{v}(t)$. The counting processes which count the cumulative occurred adult female mosquitoes who died up to time $t$ are

$$
P_{S M}^{V}\left(\int_{0}^{t} \mu_{v}(r) S_{v}(r) d r\right) \text { and } P_{I M}^{V}\left(\int_{0}^{t} \mu_{v}(r) I_{v}(r) d r\right)
$$

where $\mu_{v}(t)$ is a positive, continuous function $\tau$-periodic in time $\mathrm{t}$ for some $\tau>0,\left(P_{S M}^{V}(t)\right)_{t \geq 0}$ and $\left(P_{I M}^{V}(t)\right)_{t \geq 0}$ are standard Poisson processes.

All the considered standard Poisson processes $\left(P_{S I}^{H}(t)\right)_{t \geq 0},\left(P_{S I}^{V}(t)\right)_{t \geq 0},\left(P_{I M_{n}}^{H}(t)\right)_{t \geq 0}, \quad\left(P_{I M_{m}}^{H}(t)\right)_{t \geq 0}$, $\left(P_{I G}^{H}(t)\right)_{t \geq 0},\left(P_{L N}^{V}(t)\right)_{t \geq 0}, \quad\left(P_{S M}^{V}(t)\right)_{t \geq 0},\left(P_{I M}^{V}(t)\right)_{t \geq 0},\left(P_{S N}^{V}(t)\right)_{t \geq .},\left(P_{L_{v} M_{n}}^{V}(t)\right)$ and $\left(P_{L_{v} M_{p}}^{V}(t)\right)$ are mutually independent.

Define by $X(t)=\left(I_{h}(t), L_{v}(t), S_{v}(t), I_{v}(t)\right)$ the $\mathbb{R}^{4}$-vector which gives the state of the stochastic process of the dynamic of malaria at time $t$. Despite the continuous positive functions are $\tau$-periodic, they introduse in the model the external randomness of the environment. Then, the stochastic dynamics of malaria is described by the following stochastic differential equations driven by general counting processes (SDEsGp) 


$$
\begin{aligned}
X(t) & =X(0)+P_{S I}^{H}\left(\int_{0}^{t} c \tilde{\beta}_{v}(r) \frac{\ell S_{h}(r) I_{v}(r)}{\ell S_{h}(r)+p I_{h}(r)} d r\right)\left(\begin{array}{c}
1 \\
0 \\
0 \\
0
\end{array}\right)+P_{I G}^{H}\left(\rho \int_{0}^{t} I_{h}(r) d r\right)\left(\begin{array}{c}
-1 \\
0 \\
0 \\
0
\end{array}\right) \\
& +P_{I M_{n}}^{H}\left(d_{h} \int_{0}^{t} I_{h}(r) d r\right)\left(\begin{array}{c}
-1 \\
0 \\
0 \\
0
\end{array}\right)+P_{I M_{m}}^{H}\left(\delta_{h} \int_{0}^{t} I_{h}(r) d r\right)\left(\begin{array}{c}
-1 \\
0 \\
0 \\
0
\end{array}\right) \\
& +P_{L_{v} N}^{V}\left(\int_{0}^{t} \tilde{\lambda}_{L}(r) N_{v}(r) d r\right)\left(\begin{array}{c}
0 \\
1 \\
0 \\
0
\end{array}\right)+P_{S N}^{V}\left(\int_{0}^{t} \tilde{\lambda}_{v}(r) L_{v}(r) d r\right)\left(\begin{array}{c}
0 \\
-1 \\
1 \\
0
\end{array}\right) \\
& +P_{L_{v} M_{n}}^{V}\left(\int_{0}^{t} \tilde{\mu}_{L}(r) L_{v}(r) d r\right)\left(\begin{array}{c}
0 \\
-1 \\
0 \\
0
\end{array}\right)+P_{L_{v} M_{p}}^{V}\left(\int_{0}^{t} \alpha L_{v}^{2}(r) d r\right)\left(\begin{array}{c}
0 \\
-1 \\
0 \\
0
\end{array}\right) \\
& +P_{S I}^{V}\left(\int_{0}^{t} b \tilde{\beta}_{v}(r) \frac{p I_{h}(r) S_{v}(r)}{\ell S_{h}(r)+p I_{h}(r)} d r\right)\left(\begin{array}{c}
0 \\
0 \\
-1 \\
1
\end{array}\right)+P_{S M}^{V}\left(\int_{0}^{t} \tilde{\mu}_{v}(r) S_{v}(r) d r\right)\left(\begin{array}{c}
0 \\
0 \\
-1 \\
0
\end{array}\right) \\
& +P_{I M}^{V}\left(\int_{0}^{t} \tilde{\mu}_{v}(r) I_{v}(r) d r\right)\left(\begin{array}{c}
0 \\
0 \\
0 \\
-1
\end{array}\right),
\end{aligned}
$$

where the $\mathbb{R}^{4}$-vector $(X(t))_{t \geq 0}$ determine the born, the infection, the recovery and the death processes respectivelly of humans and mosquitoes populations in random environment.

\subsection{Example}

To illustrate our main results we consider an exemple based on actual data. We study Malaria transmission case in Abidjan based on average monthly temperature data from 1999 to 2016. In 2018, 6 countries accounted for more than half of all malaria cases worlwide and Côte d'Ivoire is in fourth place behind Nigeria (25\%), the Democratic Republic of Congo (12\%), Uganda (5\%) with (4\%) according to the latest World Malaria report released in December 2019. Related to this report children under 5 years of age are the most vulnerable group affected by malaria; in 2018, they accounted for (67\%) (272 000) of all malaria deaths worldwide. This represents a major problem of public health for affected countries. Based on data from Côte d'Ivoire, we got actual data to have good estimations of environmental parameters. Then we examine the existence and uniqueness of stochastic periodic solutions for the following associate stochastic differential equation with Poisson random measure

$$
\begin{aligned}
\tilde{Z}(t) & =\tilde{Z}(0)+\int_{0}^{t} \frac{1}{K} G(r, K \tilde{Z}(r)) d r+\sum_{l \in \mathcal{H}} \frac{h_{l}}{K} \int_{0}^{t} \int_{0}^{\infty} \mathbf{1}_{\left\{u \leq \tilde{\lambda}_{l}\left(t, K \tilde{Z}\left(s^{-}\right)\right)\right\}} \tilde{Q}_{l}^{H}(d s, d u) \\
& +\int_{0}^{t} \frac{1}{K} H(r, K \tilde{Z}(r)) d r+\sum_{j \in \mathcal{V}} \frac{h_{J}}{K} \int_{0}^{t} \int_{0}^{\infty} \mathbf{1}_{\left\{u \leq \tilde{\lambda}_{\jmath}\left(t, K \tilde{Z}\left(s^{-}\right)\right)\right\}} \tilde{Q}_{J}^{V}(d s, d u)
\end{aligned}
$$

where $\mathcal{H}=\left\{S I, I G, I M_{n}, I M_{m}\right\}$ and $\mathcal{V}=\left\{L_{v} N, S N, L_{v} M_{n}, L_{v} M_{p}, S I, S M, I M\right\}$.

First, we need to estimate the constant parameter values related to human population in Cote d'Ivoire. Abidjan has a population of $5,200,000$

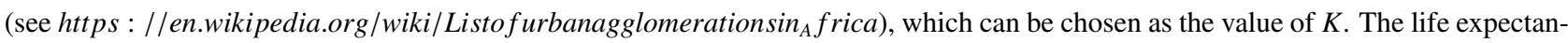
cy in Cote d'Ivoire is 57,422 years (see https : //data.worldbank.org/indicator/S P.DYN.LE00.IN?locations $=$ CI).

Taking this number, we estimate the human natural death rate as $: d_{h}=\frac{1}{57,422 \times 12}=0,00145 \mathrm{month}^{-1}$. The values $p$ and $\ell$ are probabilities and may vary from 0 to 1 with $p \geq \ell$ (Chamchod F, Britton NF, 2011), (Lacroix R and all, 2005).

Environment parameters such as temperature, play a major role in the life cycle of mosquitoes, we evaluate the periodic fonctions relative to mosquitoes population by using the monthly mean temperature of Abidjan from 1991 to 2016 (see https://climateknowledgeportal. worldbank.org/country/cote-divoire/climate-data-historical) as show in the following table. 
Table 1. Monthly mean temperatures for Abidjan (in ${ }^{\circ} \mathrm{C}$ )

\begin{tabular}{lccccccc}
\hline Month & January & February & March & April & May & June & July \\
Temperature & 26.17 & 28.20 & 28.98 & 28.49 & 27.67 & 26.33 & 25.26 \\
Month & August & September & October & November & December & & \\
Temperature & 25.02 & 25.56 & 26.25 & 26.70 & 25.99 & & \\
\hline
\end{tabular}

From data for the Wordbank obtained from Climate change knowledge, the monthly mean temperature of Abidjan is very close to those of Port Harcourt from 1990 to 2012 for which we have good estimation of those periodic fonctions. We choose the same approximate periodic functions whithout loss of generality.

According to Paaijmans and all (Paaijmans, K.P. and all, 2009), Wang, X and Zaho, X.Q (Wang, X and Zaho, X.Q, 2017); the natural biting rate of mosquitoes in Abidjan is approximate by

$$
\begin{aligned}
\beta_{v}(t) & =0.1554 \cos (\pi t / 6)+0.9065 \sin (\pi t / 6)-0.2284 \cos (2 \pi t / 6)-0.2764 \sin (2 \pi t / 6) \\
& -0.0578 \cos (3 \pi t / 6)-0.1473 \sin (3 \pi t / 6)-0.0208 \cos (4 \pi t / 6)-0.1573 \sin (4 \pi t / 6) \\
& -0.0118 \cos (5 \pi t / 6)-0.0510 \sin (5 \pi t / 6)+9.6794 \text { month }^{-1} .
\end{aligned}
$$

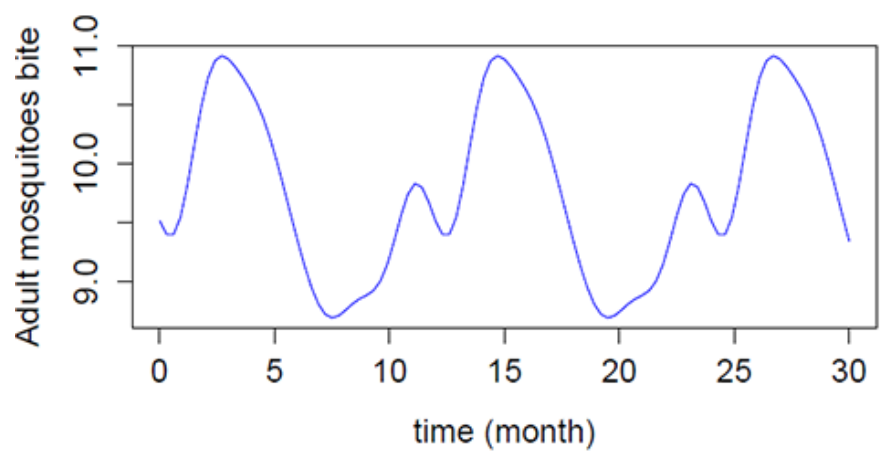

Figure 1. Number of bites of adults mosquitoes

It follow from Wang, X and Zaho, X.Q (Wang, X and Zaho, X.Q, 2017) and Rubel, F and all (Rubel, F. and all, 2008) the birth rates of juvenile and adult mosquitoes and the death rate of juvenile mosquitoes in Abidjan is approximate by

$$
\lambda_{L}(t)=2,325 \beta_{v}(t), \quad \lambda_{v}(t)=0,2325 \beta_{v}(t)
$$

and

$$
\begin{aligned}
\mu_{L}(t) & =0.2240 \cos (\pi t / 6)+1.5699 \sin (\pi t / 6)-0.4849 \cos (2 \pi t / 6)-0.4268 \sin (2 \pi t / 6) \\
& -0.0835 \cos (3 \pi t / 6)-0.3016 \sin (3 \pi t / 6)-0.0210 \cos (4 \pi t / 6)-0.2684 \sin (4 \pi t / 6) \\
& -0.0051 \cos (5 \pi t / 6)-0.0845 \sin (5 \pi t / 6)+9.0288 m^{m o n t h} h^{-1} .
\end{aligned}
$$

According to Wang, X and Zaho, X.Q (Wang, X and Zaho, X.Q, 2017), Martens and al ( Martens, P. and all, 1995) and NgarakanaGwasira and all (Ngarakana-Gwasira, E.T. and all, 2014), the death rate of adult mosquitoes in Abidjan is approximate by

$$
\begin{aligned}
\mu_{v}(r) & =0.0168 \cos (\pi t / 6)+0.1406 \sin (\pi t / 6)-0.0503 \cos (2 \pi t / 6)-0.0343 \sin (2 \pi t / 6) \\
& -0.0064 \cos (3 \pi t / 6)-0.0307 \sin (3 \pi t / 6)-0.0003 \cos (4 \pi t / 6)-0.0235 \sin (4 \pi t / 6) \\
& -0.0007 \cos (5 \pi t / 6)-0.0070 \sin (5 \pi t / 6)+3.3136 m^{m o n t h} h^{-1} .
\end{aligned}
$$




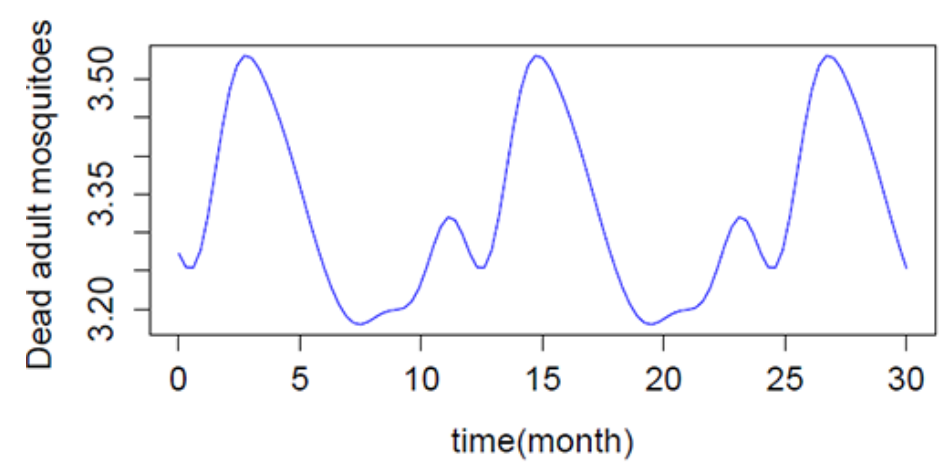

Figure 2. Number of dead adults mosquitoes

All the considered functions are continous functions in time $t$, the natural biting rate of mosquitoes, the birth rates of juvenile and adult mosquitoes, the death rate of juvenile mosquitoes and the death rate of adult mosquitoes are periodic continuous functions of period 12 months. We can notice that $\frac{1}{K} F(t, K \tilde{Z}(t))=\frac{1}{K}[G(t, K \tilde{Z}(t))+H(t, K \tilde{Z}(t))]$ does not satisfy condition (13), the linear growth condition; but for any real number $T \geq t>0$ and $n \geq 1$ the condition (20) is satisfy. Define the Lyapunov functions by $V(t, \tilde{Z}(t))=W(t, \tilde{Z}(t))=|\tilde{Z}(t)|^{2}$, where $|\tilde{Z}(t)|^{2}=\left|\tilde{I}_{h}(t)\right|^{2}+\left|\tilde{L}_{v}(t)\right|^{2}+\left|\tilde{S}_{h}(v)(t)\right|^{2}+\left|\tilde{I}_{v}(t)\right|^{2}$. Applaying Itô's formula, we proove next that all the existence conditions are satisfy and the limit process $x(t)$ in Eq. (5) exist and has the following dynamics

Sh

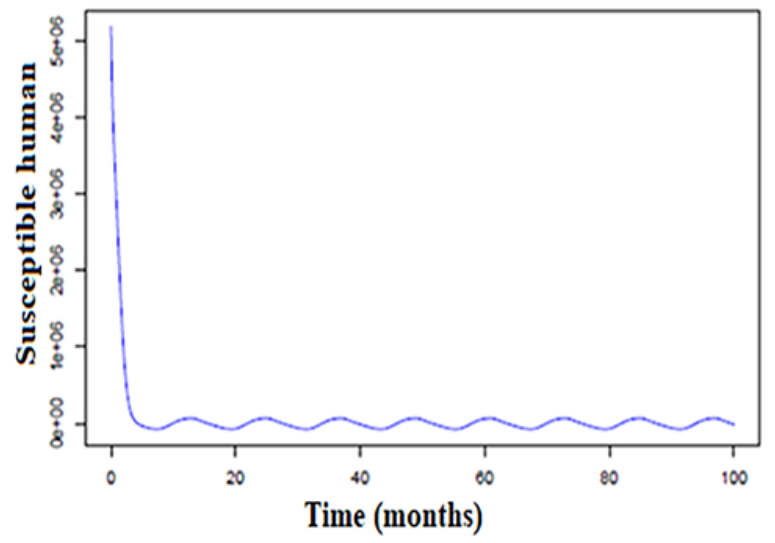

Figure 3. Simulations of the limit model Eq. (5) as a periodic function of time plotting the number of Susceptibles humans

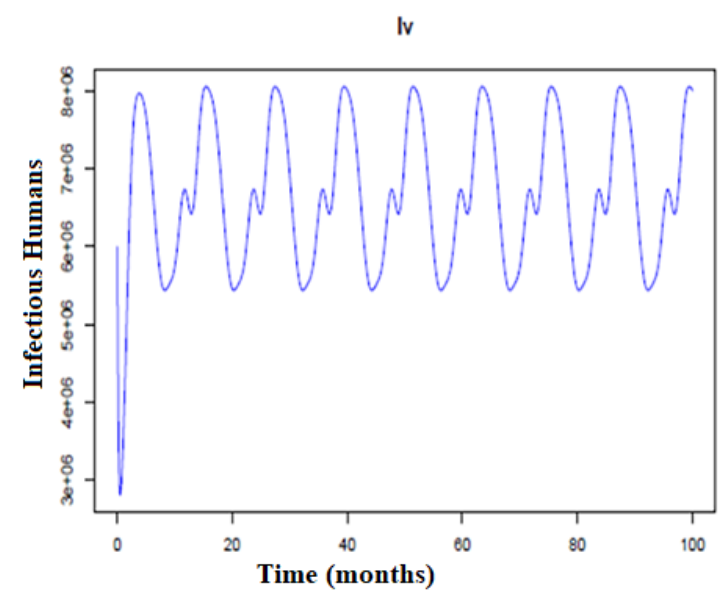

Figure 4. Simulations of the limit model Eq. (5) as a periodic function of time plotting the number of infected humans in 100 months 
LV

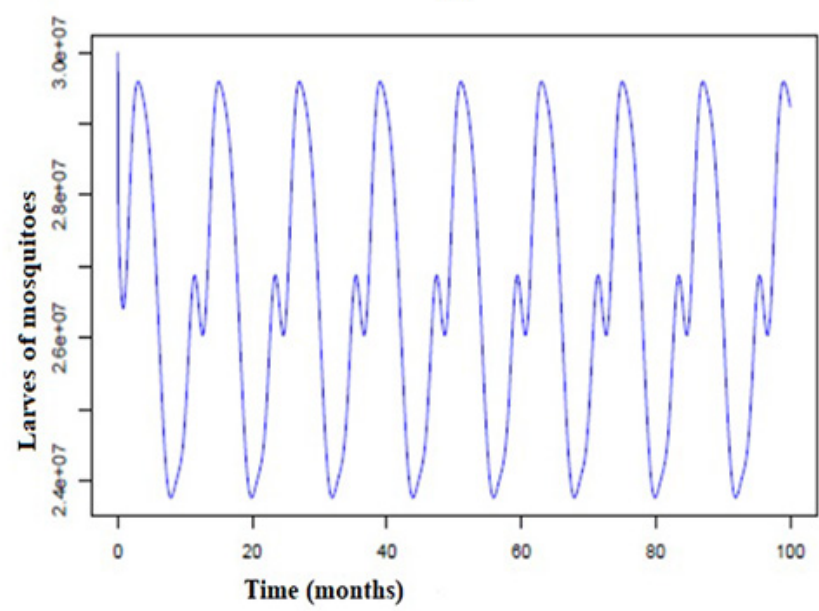

Figure 5. Simulations of the limit model (5) as a periodic function of time plotting the number of Larves of mosquitoes in 100 months

Sv

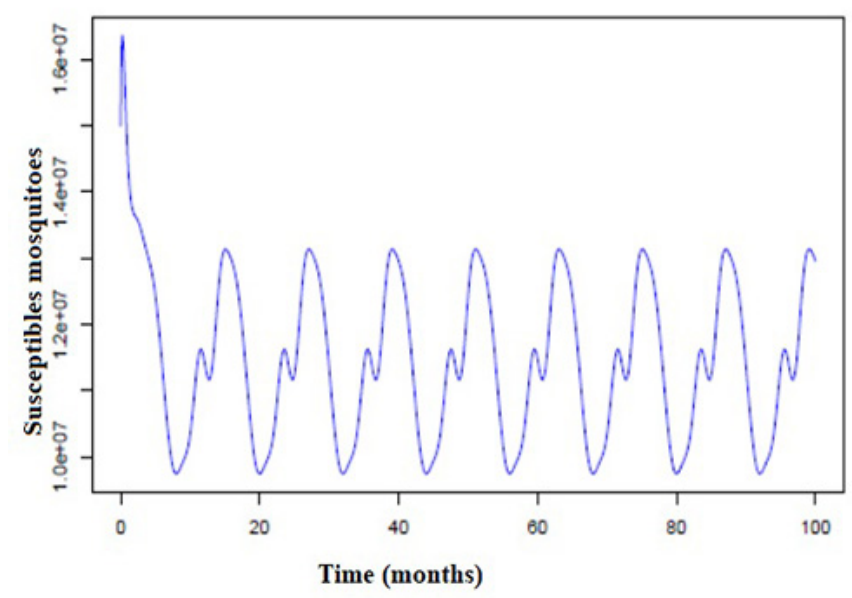

Figure 6. Simulations of the limit model Eq.(5) as a periodic function of time plotting the number of susceptible mosquitoes in 100 months

Iv

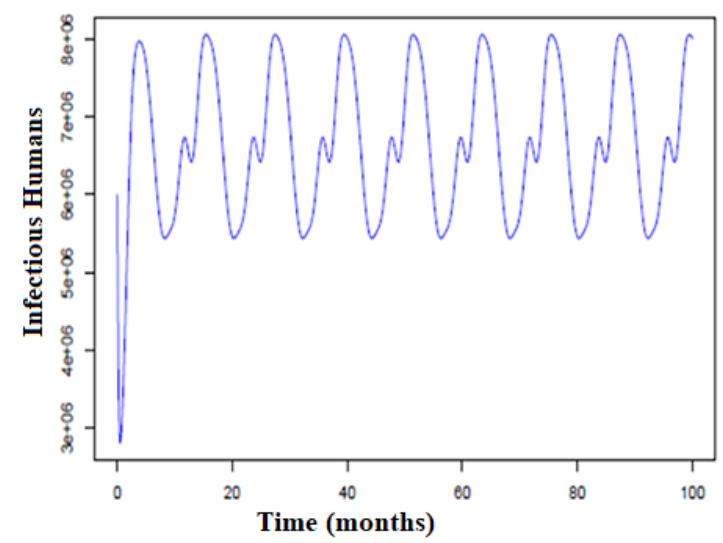

Figure 7. Simulations of the limit model Eq.(5) as a periodic function of time plotting the number of Infectious mosquitoes in 100 months 


\section{Conclusion}

We study here periodic solutions for stochastic differential equations based on counting processes with periodic stochastic intensity. Using the martingale problem, the properties of periodic stochastic processes corollated with Lyapunov's method for non-explosion of solutions of stochastic differential equations, we propose sufficient conditions to prove the existence and uniqueness of a periodic solution of the studied system. Finally, a numerical simulation on real data allowed us to consolidate the results of the mathematical analysis of the model. The analysis of the graph shows that the dynamics of malaria is sensitive to the initial conditions of the mosquito larvae, which consolidates the current strategies of control of the epidemic. In a context of climate change, the aquatic phase of mosquito development, which corresponds to the growth of mosquito larvae, deserves special attention due to its vulnerability to environmental conditions and temperature. Subsequently, a post-doctoral project on sensitivity analysis of the dynamics of the aquatic phase will allow us to quantify the temperature dependence of mosquito larval development.

\section{References}

Anderson, D. F., \& Kurtz, T. G. (2015). Stochastic analysis of biochimical systems. New York, Springer.

Billingsley, P. (1999). Convergence of probability measures. New York, John Wiley and Sons.

Britton, T., \& Pardoux, E. (2019). Stochastic epidemic models with inference. Switzerland, Springer Nature.

Chamchod, F., \& Britton N. F. (2011). Analysis of a vector bias model on malaria transmission. PLoS Biol., 3, 298. https://doi.org/10.1007/s11538-010-9545-0

Gabeli, F. (2015). Analysis of computer and communitation networks. New York, Springer

Guy, R. (2014). Approximation of epidemic model by diffusion processes and their statistical inference. J. Maths. Biol., 70, $621-646$.

Kurtz, T. G. (1980). Representation of Markov processes as multiparameter time changes. The anals of Probability, 8, $682-715$.

Kurtz, T. G. (1982). Representation and approximation of counting processes. Madison, Wisconsin 53706 USA.

Lacroix, R. (2005). Malaria infection increases attractiveness of humans to mosquitoes. PLoS Biol., 3, 298. https://doi.org/10.1371/journal.pbio.0030298

Li, D., \& Xu, D. (2013). Periodic solutions of stochastic delay differential equations and applications to logistic equation and neural networks. J. Korean Math. Soc., 50, 1165-1181. https://doi.org/10.4134/JKMS.2013.50.6.1165

Lindvall, T. (1973). Weak convergence of probability measures and random functions in the function space D. J. Appl. Prob., 10, 109-121. https://doi.org/10.2307/3212499

Luo, Q., Mao, X., \& Shen, Y. (2011). Generalised theory on asymptotic stability and boundedness of stochastic functional differential equations. Automatica, 47(9), 2075-2081. https://doi.org/10.1016/j.automatica.2011.06.014

Mandelbaum, A. (1998). Strong approximations for Markovian service networks. Queuening Systems, 30, $149-201$. https://doi.org/10.1023/A:1019112920622

Mao, X. R. (2007). Stochastic differential equations and applications (2rd ed.). Horwood, Chichester.

Martens, P. (1995). Potential impact of global climate change on malaria risk. Environ Health Perspect, 103, 458-464. https://doi.org/10.1289/ehp.95103458

Ngarakana-Gwasira, E. T. (2014). Assessing the impact of temperature on malaria transmission dynamics. Afr Mat., 25, $1095-1112$. https://doi.org/10.1007/s13370-013-0178

Paaijmans, K. P., Cator, L. J., \& Thomas, M. B. (2013). Temperature-dependent pre-bloodmeal period and temperature-driven asynchrony between parasite development and mosquito biting rate reduce malaria transmission intensity. PloS one, 8(1), e55777. https://doi.org/10.1371/journal.pone.0055777

Rubel, F. (2008). Explaining Usutu virus dynamics in Austria: model development and calibration. Prev Vet Med., 85, $166-186$. https://doi.org/10.1016/j.prevetmed.2008.01.006

Teng, L. Y. (2014). On solvability of neutral stochastic fonctional differential equations with infinite delay. Commun. Appl. Anal., 18, 325-344.

Wang, X., \& Zhao, X. Q. (2018). A climate-based malaria model with the use of bed nets. Journal of mathematical biology, 77(1), 1-25.https://doi.org/10.1007/s00285-017-1183-9.

Zhang, X., Wang, K., \& Li, D. (2015). Stochastic periodic solutions of stochastic differential equations driven by Lvy process. Journal of Mathematical Analysis and Applications, 430(1), 231-242. https://doi.org/10.1016/j.jmaa.2015.04.090

\section{Copyrights}

Copyright for this article is retained by the author(s), with first publication rights granted to the journal.

This is an open-access article distributed under the terms and conditions of the Creative Commons Attribution license (http://creativecommons.org/licenses/by/4.0/). 